\title{
Aumento da brotação de gemas na macieira cv. Maxi gala com a aplicação sequencial de indutores de brotação
}

\author{
José Luiz Petri', André Amarildo Sezerino² e Cristhian Leonardo Fenili³
}

Resumo-O objetivo do trabalho foi verificar o efeito da reaplicação dos indutores de brotação em floração, brotação e produção da macieira 'Maxi Gala'. O experimento foi desenvolvido no pomar experimental localizado no município de Caçador, durante os ciclos de 2016/2017 a 2019/2020. Os tratamentos consistiram em combinações de óleo mineral e cianamida hidrogenada, aplicados nos estádios B (gema inchada) e C (ponta verde), e a reaplicação foi feita de 7 a 14 dias após a primeira aplicação. Foram realizadas a avaliação da fenologia, da brotação de gemas axilares e terminais, da frutificação efetiva, da produção por planta e da massa média dos frutos. Os tratamentos aplicados no estádio B propiciaram um maior adiantamento dos eventos fenológicos em relação às aplicações no estádio C. A brotação das gemas axilares e dos terminais foi maior em todos os tratamentos com indutores de brotação em relação ao tratamento testemunha. Em alguns ciclos, a aplicação sequencial de indutores de brotação aumentou a brotação de gemas axilares e terminais em relação ao tratamento de uma única aplicação. A frutificação efetiva das plantas tratadas com aplicação sequencial de indutores de brotação não foi reduzida em relação ao tratamento com uma única aplicação. Alguns tratamentos com reaplicação melhoraram a produtividade das macieiras. A reaplicação de indutores de brotação aumentou a massa média dos frutos.

Termos para Indexação: Malus domestica; Quebra de dormência; Fenologia

\section{Increase of bud sprouting in apple cv. Maxi gala with sequential application of bud breaking}

Abstract - The objective of the work was to verify the effect of reapplying bud breakers on flowering, bud burst and production of the 'Maxi Gala' apple tree. The experiment was carried out in an experimental orchard located in the municipality of Caçador, during the seasons $2016 / 2017$ up to $2019 / 2020$. The treatments consisted of combinations of mineral oil and hydrogenated cyanamide, applied at phenological stages B (swollen bud) and C (green tip), and those with reapplication, from 7 to 14 days after the first application. Phenology was evaluated, bud burst of axillary and terminal buds, fruit set, production per tree and average fruit mass. The treatments applied in stage B provided a greater advance phenological events in relation to the applications in stage $\mathrm{C}$. The bud burst of axillary and terminal buds was higher in all treatments compared to the control treatment in the four years evaluated. In some seasons, the sequential application of bud breakers increased the axillary and terminal bud burst in relation to the single application standard treatment. The fruit set of treated plants with sequential application of bud breakers was not reduced compared to treatment with a single application. Some treatments with reapplication improved the productivity of the apple trees. The reapplication of bud breakers increased the average fruit mass.

Index Terms: Malus domestica; Bud breaking; Phenology.

\section{Introdução}

No Sul do Brasil, a maior parte do cultivo da macieira está localizada em áreas onde a necessidade em frio dos principais cultivares não é plenamente satisfeita. Nesse sentido, em condições de invernos mais amenos, diversas práticas para a superação artificial da dormência têm sido utilizadas com o objetivo de amenizar alguns dos problemas decorrentes da deficiência de frio, proporcionando brotação e floração adequadas nas principais espécies de clima temperado (PETRI et al., 2008). Em condições de insuficiência em frio hibernal, fruteiras de clima temperado apresentam anormalidades em relação à brotação, tendo repercussão durante o ciclo vegetativo e acarretando a redução tanto da produtividade quanto da qualidade dos frutos produzidos (PETRI et al., 2010). Tanto a regularidade quanto a quantidade de frio são indispensáveis para a superação natural da dormência. Entre as práticas que mais respondem à indução da brotação nestas condições, destaca-se o uso de produtos químicos, chamados de indutores de brotação. Baixas taxas de brotação reduzem a área foliar total, diminuindo desse modo a fotossíntese total da plan- ta. Esse fato pode levar a uma carência na nutrição da planta, com consequências das mais diversas, mas complementares, como baixa frutificação efetiva e redução do ciclo entre a floração e a maturação.

No Brasil, os primeiros trabalhos com indução da brotação em macieira são de 1972 (PETRI, et al., 2012). A partir da década de 80, o óleo mineral (OM), a cianamida hidrogenada $(\mathrm{CH})$ e a associação de ambos tornaram-se os produtos mais utilizados na indução da brotação das fruteiras de clima temperado (HAWERROTH et al., 2010). Uma nova geração de produtos foi desenvolvida a

Recebido em 2/3/2021. Aceito para publicação em 3/5/2021.

http//:dx.doi.org/10.52945/rac.v34i2.1136

${ }^{1}$ Engenheiro-agrônomo, M.Sc. (aposentado), Estação Experimental de Caçador (Epagri/EECd), C.P. 591, 89501-032 Caçador, SC e-mail: petri@epagri.sc.gov.

br

${ }^{2}$ Engenheiro-agrônomo, Dr., Epagri/EECd, e-mail: andresezerino@epagri.sc.gov.br

${ }^{3}$ Engenheiro-agrônomo, M.Sc., Udesc/Centro de Ciências Agroveterinárias (CAV), Lages, SC, e-mail: cristhianfenili@hotmail.com 
partir dos anos 2000, devido às restrições de uso da cianamida hidrogenada, que possui alta toxicidade (HERNÁNDEZ \& CRAIG, 2011). Novas moléculas, que apresentam em sua composição nitrogênio inorgânico, aminoácidos, polissacarídeos, ácido glutâmico e nutrientes minerais, como Erger ${ }^{\circ}$, Syncron ${ }^{\circ}$, Thidiazuron (TDZ), Siberio e Bluprins ${ }^{\circ}$, em mistura com nitrato de cálcio $\left(\mathrm{Ca}\left(\mathrm{NO}_{3}\right)_{2}\right)$ ou óleo mineral, têm efeito na indução da brotação da macieira, apresentando muitas vezes resultados similares aos do tratamento padrão de óleo mineral mais cianamida hidrogenada (PASA et al., 2018; FENILI et al., 2018; HAWERROTH et al., 2010).

Com os novos sistemas de plantio em alta densidade, tanto na formação da planta quanto na fase de frutificação, é importante que se obtenha o máximo possível de brotação para evitar a falta de ramos de produção em consequência da brotação deficiente. Em algumas situações pode haver grande variabilidade na resposta das plantas à aplicação dos indutores, podendo haver brotação insuficiente, sendo necessária nova intervenção. A aplicação sequencial consiste na reaplicação do tratamento e pode ser realizada na planta toda ou somente na parte superior da copa, uniformizando a brotação e a floração. Todavia, a reaplicação de indutores de brotação ainda é pouco estudada e seus efeitos ainda são desconhecidos.

O objetivo do trabalho foi verificar o efeito da reaplicação dos indutores de brotação na floração, na brotação e na produção da macieira 'Maxi Gala'.

\section{Material e métodos}

$\mathrm{O}$ experimento foi desenvolvido em pomar experimental localizado no município de Caçador, SC (latitude $26^{\circ} 46^{\prime} \mathrm{S}$, longitude $51^{\circ} \mathrm{W}$, altitude 960 metros), durante os ciclos de 2016/2017 a 2019/2020. Segundo classificação de Köppen, o clima na região de cultivo é classificado como $\mathrm{Cfb}$ - temperado constantemente úmido, com verão ameno. A média da precipitação pluvial anual é de $1653,2 \mathrm{~mm}$ e a umidade relativa do ar média é de $77,9 \%$. Segundo Petri et al. (2010), a média do acúmulo de frio durante o período de outono e de inverno é de 928 unidades de frio, modelo Carolina do Norte Modificado (EBERT, et al., 1986), sendo que, nos in- vernos de 2016, 2017, 2018 e 2019, o acúmulo destas unidades foi de 1.305, $628,1.170$ e 593 , respectivamente.

Utilizaram-se plantas de 10 anos de idade do cv. Maxi Gala, enxertado sobre o porta-enxerto Marubakaido com interenxerto de M-9. A densidade de plantio no pomar utilizado foi de 2.500 plantas $\mathrm{ha}^{-1}$, com espaçamento de $4 \mathrm{~m}$ entre linhas e $1 \mathrm{~m}$ entre plantas, sendo as plantas manejadas no sistema de condução em líder central. $O$ esquema de polinização adotado baseava-se na utilização de dois cultivares produtores, sendo Maxi Gala o polinizador do cv. Fuji Suprema e vice-versa. A disposição das plantas foi em filas inteiras, com uma planta polinizadora a cada quinze plantas produtoras e duas filas de 'Maxi Gala' por duas de 'Fuji Suprema'. Desde a implantação do experimento até o término da realização deste estudo, o pomar foi conduzido de acordo com as práticas de manejo recomendadas no sistema de produção da macieira (SEZERINO, 2018).

O delineamento experimental utilizado foi em blocos casualizados, com cinco repetições, sendo cada unidade composta por uma planta. Os tratamentos são apresentados na Tabela 1. A aplicação dos indutores de brotação foi realizada através de aspersão com pulverizador costal motorizado, com um volume médio equivalente à $1000 \mathrm{~L} \mathrm{ha}^{-1}$. Os tratamentos foram aplicados no estádio B (gema inchada ou ponta prata) e C (ponta verde) e a reaplicação foi feita de 7 a 14 dias após a primeira aplicação (Tabela 1). Foi realizada a avaliação da fenologia dos estádios C-C3 (ponta verde até $1,3 \mathrm{~cm}$ verde), do início da brota- ção e início, plena e final da floração, da taxa de brotação de gemas axilares e de gemas terminais, da frutificação efetiva, da produção por planta, do número de frutos por planta e do peso médio dos frutos.

O início de floração foi considerado quando as plantas estavam com $5 \%$ de flores abertas, a plena floração quando verificado que mais de $80 \%$ das flores estavam abertas e o fim de floração foi apontado quando as últimas flores estavam abertas. A brotação de gemas axilares foi obtida da contagem de gemas brotadas e não brotadas em cinco brindilas previamente selecionadas, localizadas no terço médio da planta. Para estimativa da porcentagem de brotação de gemas terminais, foi selecionada uma ramificação lateral representativa (com média de 100 gemas) de cada planta e realizada a contagem de gemas terminais brotadas e não brotadas. A frutificação efetiva foi obtida da relação entre o número de frutos contados logo após o pegamento de frutos (estádio J), e número de cachos florais contados durante a plena floração (estádio F2) ([número de frutos/cachos florais] $x 100)$, sendo as contagens realizadas na mesma ramificação lateral utilizada para estimar a brotação de gemas terminais. O número de frutos por planta e a produção por planta foram obtidos através da colheita total dos frutos quando da maturação dos mesmos. A colheita foi realizada em duas datas, de acordo com a data de aplicação dos indutores de brotação.

Os dados obtidos foram submetidos à análise da variância, cujas variáveis

Tabela 1. Tratamentos e estádio de aplicação Table 1. Treatments and application stage

\begin{tabular}{|c|c|c|}
\hline Tratamento & Aplicação & Reaplicação \\
\hline 1.Testemunha & - & - \\
\hline 2. $\mathrm{OM} 3,5 \%+\mathrm{CH} 0,7 \%$ & Estádio B & - \\
\hline 3. (OM 3,5\% + CH 0,7\%)+(OM 3,5\%+CH 0,7\%) & Estádio B & $7 \mathrm{DA}$ \\
\hline 4. $(\mathrm{OM} 3,5 \%+\mathrm{CH} 0,7 \%)+(\mathrm{OM} 3,5 \%+\mathrm{CH} 0,7 \%)$ & Estádio B & $14 \mathrm{DA}$ \\
\hline 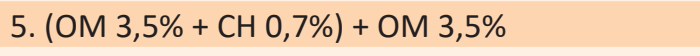 & Estádio B & $7 \mathrm{DA}$ \\
\hline 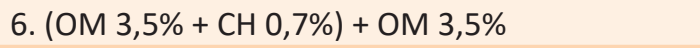 & Estádio B & $14 \mathrm{DA}$ \\
\hline 7. $(\mathrm{OM} 3,5 \%+\mathrm{CH} 0,7 \%)+\mathrm{CH} 1,0 \%$ & Estádio B & $7 \mathrm{DA}$ \\
\hline 8. $(\mathrm{OM} 3,5 \%+\mathrm{CH} 0,7 \%)+\mathrm{CH} 1,0 \%$ & Estádio B & $14 \mathrm{DA}$ \\
\hline 9. (OM $3,5 \%+\mathrm{CH} 0,7 \%)+\mathrm{CH} 0,7 \%$ & Estádio C & $5 \mathrm{DA}$ \\
\hline 10. (OM 3,5\% + CH 0,7\%) + (OM 3,5\%+CH 0,7\%) & Estádio C & $10 \mathrm{DA}$ \\
\hline 11. $(\mathrm{OM} 3,5 \%+\mathrm{CH} 0,7 \%)+\mathrm{OM} 3,5 \%$ & Estádio C & $10 \mathrm{DA}$ \\
\hline
\end{tabular}

$\mathrm{OM}=$ Óleo mineral; $\mathrm{CH}=$ Cianamida Hidrogenada (Dormex $\left.{ }^{\circledast}\right) ; \mathrm{DA}=$ Dias após. 
significativas $(p<0,05)$ tiveram as médias comparadas pelo teste Scott-Knott a $5 \%$ de probabilidade de erro. Dados em porcentagem foram transformados pela fórmula arco seno $[(x+1) / 100]^{1 / 2}$ antes de serem submetidos à Anova. Os procedimentos de análise foram realizados por meio do programa Sisvar, versão 5.6 (FERREIRA, 2010).

\section{Resultados e discussão}

A fenologia foi adiantada em relação ao tratamento testemunha com a aplicação dos indutores de brotação, independente do estádio de aplicação e da aplicação sequencial (Figura 1). Quanto ao estádio de aplicação, os tratamentos aplicados no estádio B propiciaram um maior adiantamento em relação às aplicações no estádio C no cultivar Maxi Gala. Quanto à aplicação sequencial, foi observado um pequeno efeito em comparação a uma única aplicação, o mesmo ocorrendo com a reaplicação somente de Óleo mineral (OM) ou Cianamida hidrogenada $(\mathrm{CH})$. O maior efeito na antecipação da floração foi com relação ao estádio fenológico da primeira aplicação, onde os tratamentos aplicados no estádio $C$ retardaram a floração em relação aos tratamentos aplicados no estádio $B$, o que confirma resultados de Petri et al. (2012). George et al. (2002) afirmam que indutores de brotação podem ser utilizados para modular a época de brotação e floração. Observa-se também uma influência do ano em relação à antecipação dos estádios fenológicos, o que pode estar relacionado à intensidade do frio (PETRI et al., 2012). As maiores antecipações da plena floração, em relação às plantas testemunhas, foram de 19, 33, 34 e 27 dias, para os anos de 2016, 2017, 2018 e 2019, respectivamente (Figura 1). Para as aplicações no estádio $B$, também mostrou tendência a adiantar os estádios fenológicos em relação aos aplicados no estádio $C$, porém menor que os observados em relação às plantas sem tratamento. Hawerroth et al. (2010) também observaram um adiantamento da floração com aplicações de OM mais $\mathrm{CH}$. Segundo Pasa et al. (2018), a antecipação da floração com indutores de brotação é maior nas aplicações antecipadas após completar a endodormência, no final do inverno. Os indutores de brotação encurtam o período entre a plena floração e o final da floração (Tabela 1). Petri et al. (2008) também verificaram que a aplicação de OM em macieiras antecipa o florescimento e reduz seu período.

Todos os tratamentos com indutores de brotação proporcionaram maiores taxas de brotação das gemas axilares comparados às macieiras que não receberam indução de brotação nos quatro anos de estudo (Tabela 2). Entre os tratamentos, destacam-se T5. [(OM 3,5\% + $\mathrm{CH}$ 0,7\% Estádio B) + (OM 3,5\% 7 DA)], T8. $[(\mathrm{OM} 3,5 \%+\mathrm{CH}$ 0,7\% Estádio $\mathrm{B})+$ ( $\mathrm{CH} 1,0 \% 14 \mathrm{DA})]$ e T9. [(OM 3,5\% + $\mathrm{CH}$ 0,7\% Estádio C) + ( $\mathrm{CH} \mathrm{0,7 \%} 5$ DA)], que nos quatro anos proporcionaram brotações axilares significativamente superiores às induzidas pelos demais tratamentos aos 30 dias após a aplicação. Estes mesmos tratamentos, aos 60 dias, proporcionaram as maiores taxas de brotação em três anos, o que demonstra que aplicação sequencial aumenta o percentual de brotação das gemas axilares. A transição entre as diferentes fases da dormência envolve aspectos genéticos, fisiológicos, bioquímicos e anatômicos (FRANCESCATTO, 2014). Estas alterações causam efeitos diferenciados, dependendo do estádio de aplicação dos tratamentos (COOKE et al., 2012). Os percentuais de brotação de gemas axilares também variaram com os anos, o que pode ser atribuído ao frio ocorrido em 2019/2020, que apresentou os menores percentuais de brotação, sendo o ano com a menor intensidade de frio no período em que foi conduzido o experimento. Pasa et al. (2018), estudando o efeito de indutores de brotação no cv. Maxi Gala em São Joaquim, SC, observaram aumento na brotação das gemas axilares com o uso dos indutores somente no ano em que fez menor intensidade de frio. A brotação das gemas axilares é importante para a formação de estruturas de frutificação para o ano seguinte e evitar falhas na formação da planta afetando o volume da copa.

Destaca-se que a taxa de brotação das gemas axilares no tratamento testemunha variou de $0,0 \%$ a $8,6 \%$ de acordo com o ano, em média $96 \%$ menos brotação de gemas em relação aos tratamentos com indutores de brotação, onde a taxa variou de $20,5 \%$ a $78,3 \%$, o que está de acordo com De Martin et al. (2017).

A brotação das gemas terminais, localizadas na extremidade das brindilas,

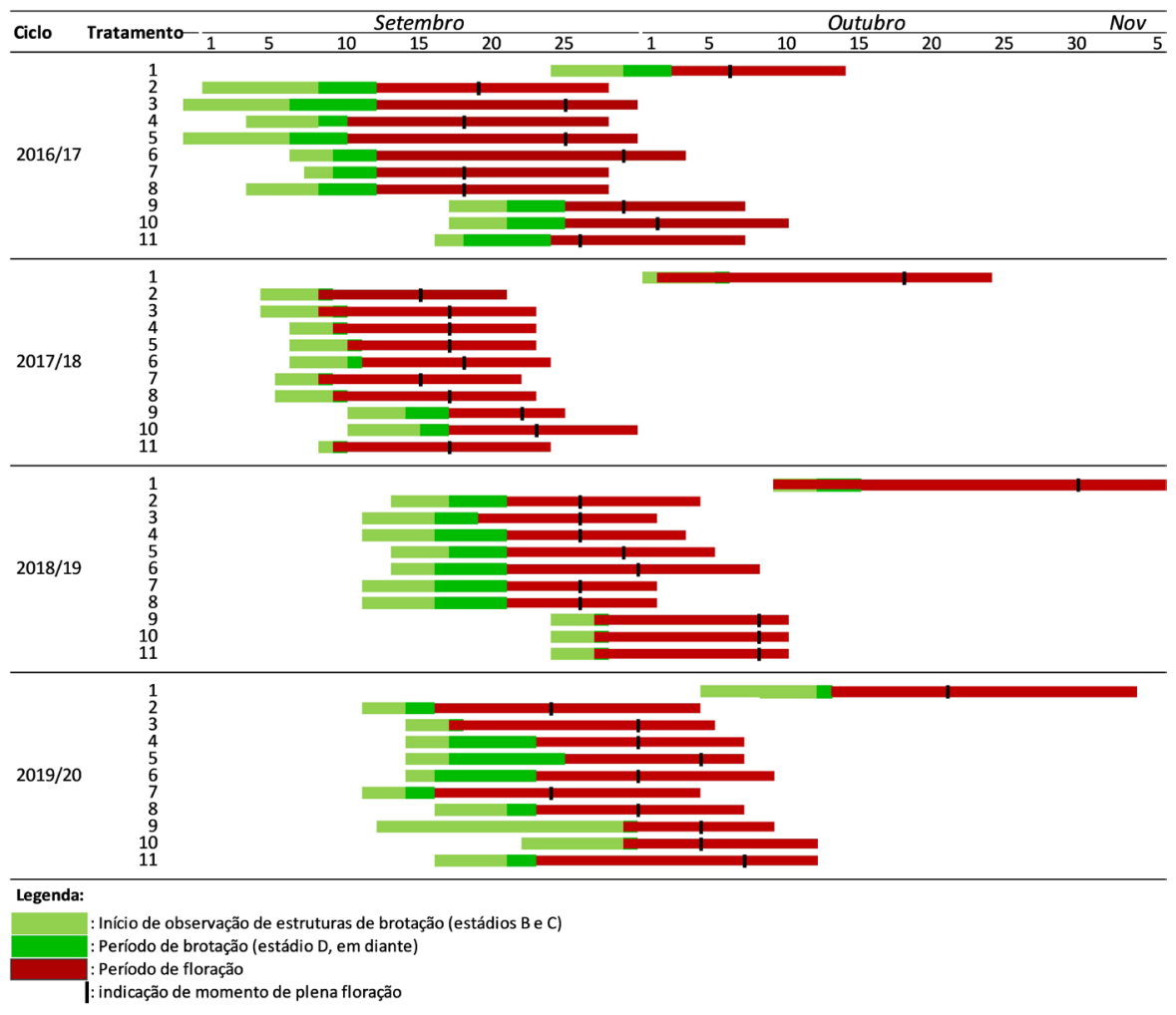

Figura 1. Evolução dos estádios fenológicos da macieira 'Maxi Gala' com diferentes tratamentos de indutores da brotação, em quatro ciclos - Caçador, SC

Figure 1. Evolution of the phenological stages of the 'Maxi Gala' apple tree with different treatments of bud breaking, in four cycles - Caçador, SC 
no cv. Maxi Gala, todos os tratamentos diferiram do tratamento testemunha aos 30 e 60 dias após a plena floração (Tabela 3). Quanto ao tratamento padrão, de uma única aplicação, foi significativamente inferior aos tratamentos de aplicação sequencial nos anos de 2018/19 e 2019/20, apresentando menor brotação de gemas terminais. Nos anos de 2017/18 e 2019/20 o tratamento T9. [(OM 3,5\% + $\mathrm{CH}$ 0,7\% Estádio C) $+(\mathrm{CH} 0,7 \% 5 \mathrm{DA})]$ foi significativamente superior aos demais tratamentos. Normalmente as gemas terminais, por serem floríferas, brotam mais que as gemas axilares, devido à menor exigência em frio e pela dominância apical (UBER et al., 2019). De acordo com Francescatto (2014), a falta de brotação das gemas axilares pode favorecer a brotação das gemas terminais e comprometer a formação de órgãos reprodutivos, favorecendo o desenvolvimento vegetativo excessivo.

O conjunto da brotação das gemas axilares e terminais evidenciam uma melhor brotação com aplicações sequenciais, havendo um incremento significativo, principalmente na brotação das gemas axilares, que são as responsáveis pela formação das estruturas de frutificação para o ano seguinte (Tabelas 1 e 2). Cabe ressaltar que não foi observado nenhum problema de queima ou desidratação de ramos e gemas em consequência da reaplicação dos indutores de brotação.

A frutificação efetiva foi reduzida no Maxi Gala em alguns tratamentos em relação ao tratamento testemunha no ano de 2016/17, porém o mesmo não ocorreu nos demais anos (dados não apresentados). Observou-se também que não houve diferenças na frutificação efetiva entre a aplicação única e as aplicações sequenciais, sendo que os mais altos percentuais de frutificação efetiva foram observados no tratamento testemunha no ano em que esta variável apresentou diferenças significativas. Não se observou redução da frutificação efetiva nos tratamentos com aplicação sequencial em relação à aplicação única, bem como entre as datas de reaplicação e as aplicações que envolvem a reaplicação com OM acrescido de $\mathrm{CH}$. A redução da frutificação efetiva pode estar relacionada à concentração da floração com o uso dos indutores de brotação, o que pode aumentar o risco de uma polinização deficiente, principalmente se as condições ambientais forem desfavoráveis ao trabalho das abelhas (HERNANDEZ \& CRAIG, 2011).

Quanto à produção por planta na Maxi Gala, foi possível observar que a realização dos tratamentos T5. [(OM $3,5 \%+\mathrm{CH} 0,7 \%$ Estádio B) + (OM 3,5\%
7 DA)], T6. [(OM 3,5\% + CH 0,7\% Estádio $\mathrm{B})+(\mathrm{OM} 3,5 \% 14 \mathrm{DA})]$ e T11. [(OM $3,5 \%+\mathrm{CH} 0,7 \%$ Estádio C) + (OM 3,5\% $10 \mathrm{DA})]$ promoveu resultados significativamente superiores ao tratamento testemunha e ao tratamento de aplicação única nos ciclos 2016/17, 2018/19, 2019/20 e na média dos quatro anos (Tabela 4). Não se observou diferença significativa quanto à reaplicação aos 7 ou 14 dias após a primeira aplicação. Quanto à reaplicação somente com OM, foram observadas maiores produtividades em macieiras tratadas com T5. [(OM $3,5 \%+\mathrm{CH} 0,7 \%$ estádio $\mathrm{B})+(\mathrm{OM} 3,5 \%$ 7DA)], T6. [(OM 3,5\% + CH 0,7\% estádio B) + (OM 3,5\% 14DA)] e T11. [(OM $3,5 \%+\mathrm{CH} 0,7 \%$ estádio $\mathrm{C})+(\mathrm{OM} 3,5 \%$ $10 \mathrm{DA})]$, em relação às tratadas com $\mathrm{OM}$ mais $\mathrm{CH}$ ou somente $\mathrm{CH}$. Estes resultados podem estar relacionados à concentração da floração nos tratamentos que envolvem a reaplicação $\mathrm{com} \mathrm{CH}$. O tratamento com maior produtividade T11. [(OM 3,5\% + CH 0,7\% estádio C) + (OM 3,5\% 10DA)] aumentou em média $78,5 \%$ a produção por planta em relação ao tratamento testemunha e 130,9\% em relação ao tratamento de aplicação única. Este mesmo comportamento foi observado quanto ao número de frutos por planta (Tabela 4).

A massa média dos frutos apresentou grande variabilidade entre os anos

Tabela 2. Brotação de gemas axilares (\%) aos 30 e aos 60 dias após a quebra da dormência de macieiras 'Maxi Gala', tratadas com diferentes indutores de brotação, em quatro ciclos - Caçador, SC

Table 2. Bud burst of axillary (\%) at 30 and 60 days after the dormancy break of 'Maxi Gala' apple trees, treated with different bud breaking, in four cycles - Caçador, SC

\begin{tabular}{|c|c|c|c|c|c|c|c|c|}
\hline \multirow{2}{*}{ Tratamento } & \multicolumn{8}{|c|}{ Brotação de gemas axilares (\%) } \\
\hline & $2016 / 17$ & $2017 / 18$ & $2018 / 19$ & $2019 / 20$ & $2016 / 17$ & $2017 / 18$ & $2018 / 19$ & $2019 / 20$ \\
\hline & \multicolumn{4}{|c|}{30 dias após a quebra de dormência } & \multicolumn{4}{|c|}{60 dias após a quebra de dormência } \\
\hline $\mathrm{T} 2$. & $50,9 \mathrm{~b}$ & $37,3 \mathrm{~b}$ & $35,9 \mathrm{~b}$ & $21,5 b$ & $54,3 \mathrm{~b}$ & $38,7 \mathrm{~b}$ & 42,6 a & $23,8 \mathrm{c}$ \\
\hline T3. & 74,0 a & $45,7 b$ & 47,3 a & $28,4 \mathrm{~b}$ & 78,3 a & $49,5 \mathrm{~b}$ & 54,0 a & $32,5 \mathrm{c}$ \\
\hline T6. & $57,5 b$ & 63,6 a & 55,3 a & $22,5 b$ & $58,6 \mathrm{~b}$ & 65,0 a & $63,4 \mathrm{a}$ & $24,4 \mathrm{c}$ \\
\hline T7. & $63,6 \mathrm{a}$ & $30,7 \mathrm{~b}$ & 47,9 a & $36,9 a$ & $68,1 \mathrm{~b}$ & $34,9 \mathrm{~b}$ & $55,5 \mathrm{a}$ & $38,5 \mathrm{~b}$ \\
\hline T8. & 68,7 a & $58,0 \mathrm{a}$ & 51,0 a & 36,9 a & 73,5 a & 60,5 a & $60,8 \mathrm{a}$ & 40,9 b \\
\hline T9. & 60,1 a & 65,5 a & $42,8 \mathrm{a}$ & 47,6 a & $75,3 \mathrm{a}$ & 66,0 a & 63,2 a & 57,2 a \\
\hline
\end{tabular}

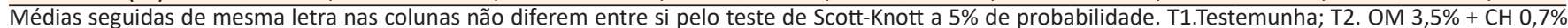

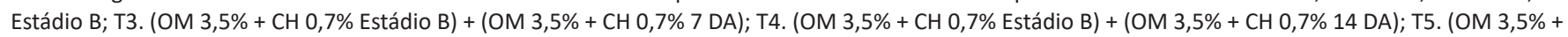

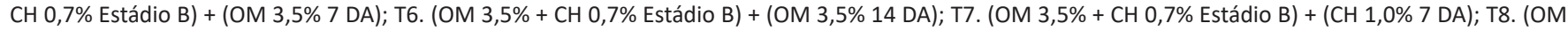

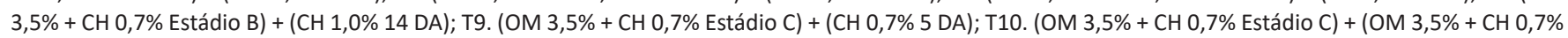
10 DA); T11. (OM 3,5\% + CH 0,7\% Estádio C) + (OM 3,5\% 10 DA). 
Tabela 3. Brotação de gemas terminais (\%) aos 30 e aos 60 dias após a quebra da dormência de macieiras 'Maxi Gala', tratadas com diferentes indutores de brotação, em quatro ciclos - Caçador, SC

Table 3. Bud burst of terminal (\%) at 30 and 60 days after the dormancy break of 'Maxi Gala' apple trees, treated with different bud breaking, in four cycles - Caçador, SC

\begin{tabular}{|c|c|c|c|c|c|c|c|c|}
\hline \multirow{2}{*}{ Tratamento } & \multicolumn{8}{|c|}{ Brotação de gemas terminais (\%) } \\
\hline & $2016 / 17$ & $2017 / 18$ & $2018 / 19$ & $2019 / 20$ & $2016 / 17$ & $2017 / 18$ & $2018 / 19$ & $2019 / 20$ \\
\hline & \multicolumn{4}{|c|}{30 dias após a quebra de dormência } & \multicolumn{4}{|c|}{60 dias após a quebra de dormência } \\
\hline T1. & $25 d$ & $4 \mathrm{~b}$ & $16 \mathrm{~b}$ & $12 \mathrm{c}$ & $74 \mathrm{c}$ & $31 \mathrm{c}$ & $32 \mathrm{c}$ & $44 d$ \\
\hline $\mathrm{T} 2$. & 97 a & 83 a & $56 \mathrm{a}$ & $71 \mathrm{~b}$ & 97 a & $91 b$ & $64 \mathrm{~b}$ & $73 \mathrm{c}$ \\
\hline T3. & $100 \mathrm{a}$ & 76 a & $87 \mathrm{a}$ & $84 \mathrm{~b}$ & $100 \mathrm{a}$ & $93 \mathrm{~b}$ & 89 a & $88 \mathrm{~b}$ \\
\hline T4. & 98 a & 89 a & 73 a & $79 \mathrm{~b}$ & $100 \mathrm{a}$ & $92 \mathrm{~b}$ & 78 a & $91 \mathrm{~b}$ \\
\hline T7. & 99 a & 73 a & 80 a & $88 \mathrm{~b}$ & 99 a & $88 b$ & 90 a & $96 \mathrm{~b}$ \\
\hline T8. & $100 \mathrm{a}$ & $84 \mathrm{a}$ & $73 a$ & $73 b$ & $100 \mathrm{a}$ & $86 b$ & 79 a & $88 b$ \\
\hline T9. & $92 b$ & 96 a & $92 \mathrm{a}$ & $100 \mathrm{a}$ & $100 \mathrm{a}$ & 98 a & $93 a$ & $100 \mathrm{a}$ \\
\hline T10. & $85 b$ & 90 a & $77 \mathrm{a}$ & $79 b$ & 99 a & $98 \mathrm{a}$ & 83 a & $86 b$ \\
\hline T11. & $74 \mathrm{c}$ & 75 a & $66 \mathrm{a}$ & $76 b$ & $92 b$ & $83 b$ & $86 a$ & $79 b$ \\
\hline
\end{tabular}

Médias seguidas de mesma letra nas colunas não diferem entre si pelo teste de Scott-Knott a 5\% de probabilidade. T1.Testemunha; T2. OM 3,5\% + CH 0,7\% Estádio B; T3. (OM 3,5\% + CH 0,7\% Estádio B) + (OM 3,5\% + CH 0,7\% 7 DA); T4. (OM 3,5\% + CH 0,7\% Estádio B) + (OM 3,5\% + CH 0,7\% 14 DA); T5. (OM 3,5\% + $\mathrm{CH}$ 0,7\% Estádio B) + (OM 3,5\% 7 DA); T6. (OM 3,5\% + CH 0,7\% Estádio B) + (OM 3,5\% 14 DA); T7. (OM 3,5\% + CH 0,7\% Estádio B) + (CH 1,0\% 7 DA); T8. (OM $3,5 \%+\mathrm{CH} 0,7 \%$ Estádio B $)+(\mathrm{CH} 1,0 \% 14 \mathrm{DA}) ; \mathrm{T} 9 .(\mathrm{OM} 3,5 \%+\mathrm{CH} 0,7 \%$ Estádio C $)+(\mathrm{CH} 0,7 \% 5 \mathrm{DA}) ; \mathrm{T} 10 .(\mathrm{OM} 3,5 \%+\mathrm{CH} 0,7 \%$ Estádio C) + (OM 3,5\% + CH 0,7\% $10 \mathrm{DA}) ; \mathrm{T} 11 .(\mathrm{OM} 3,5 \%+\mathrm{CH} 0,7 \%$ Estádio C) + (OM 3,5\% $10 \mathrm{DA})$.

estudados e geralmente foi afetada pela produção das plantas, sendo que plantas de tratamentos com maior produção apresentaram menor massa média dos frutos (Tabela 5). Apenas no ciclo 2018/2019 não ocorreram diferenças na massa média dos frutos entre os tratamentos. Já na média das quatro safras estudadas, apenas o tratamento padrão, de uma única aplicação, apresentou redução da massa média das maçãs, juntamente com o T6. [(OM 3,5\% + CH 0,7\% Estádio B) + (OM 3,5\% $14 \mathrm{DA})]$. Todavia, o T6. [(OM 3,5\% + CH 0,7\% Estádio B) + (OM 3,5\% 14 DA)], que apresentou na média uma produção por planta superior aos demais tratamentos (Tabela 4), o que pode estar atribuído à redução da massa média dos frutos.

\section{Conclusão}

A aplicação de indutores de brotação no estádio $B$ adianta os estádios fenológicos da macieira 'Maxi Gala' em relação à aplicação de indutores no estádio $C$, independentemente da reaplicação e dos intervalos de tempo testados.

A aplicação sequencial de indutores de brotação aumenta o percentual de brotação das gemas axilares e terminais da macieira 'Maxi Gala' e não reduz a frutificação efetiva em relação à aplicação única.

A reaplicação de óleo mineral isola- do na quebra de dormência da macieira 'Maxi Gala', após a primeira aplicação no estádio $\mathrm{B}$ ou $\mathrm{C}$, aumenta a produção das plantas.

A aplicação sequencial de indutores de brotação aumenta a massa média das maçãs 'Maxi Gala' comparada a uma única aplicação.

\section{Referências}

COOKE, J.E.K., ERIKSSON, M.E. JUNTTILA, $O$. The dynamic nature of bud dormancy in trees: environmental control and molecular mechanisms. Plant Cell Environ, v.35, p.1707-1728, 2012. DOI: https://doi. org/10.1111 / j.1365-3040.2012.02552.x.

DE MARTIN, M. S., PETRI, J. L., FENILI, C. L., GABARDO, G. C. Quebra da dormência de macieiras 'Daiane' pelo uso do tidiazurom. Revista de Ciências Agroveterinárias, Lages, v.16, n.2, p.81-87, 2017. DOI: https://doi. org/10.5965/223811711622017081.

EBERT, A.; PETRI, J.L.; BENDER, R.J.; BRAGA, H.J. First experiences with chill-unit models in Southern Brazil. Acta Horticulturae, Hohenheim, n.184, p. 79-86, 1986. DOI: https:// doi. org/10.17660/ActaHortic.1986.184.8.

FENILI, C.L.; PETRI, J.L.; SEZERINO, A.A.; DE MARTIN, M.S.; GABARDO, G.C.; DANIEL, E.S. Bluprins $^{\circledR}$ as Alternative Bud Break Promoter for 'Maxi Gala' and 'Fuji Suprema' Apple Trees. Journal of Experimental Agriculture International, v.26, n.2, p.1-13, 2018. DOI:
https://doi.org/10.9734/JEAI/2018/43649.

FERREIRA, D. F. Sisvar - programa estatístico. Versão 5.6 (Build 86). Lavras: Universidade Federal de Lavras, 2010.

FRANCESCATTO P. Desenvolvimento das estruturas reprodutivas da macieira (Malus domestica Borkh.) sob diferentes condições climáticas: da formação da gema à colheita dos frutos. Thesis (PhD in Plant Genetic Resources). Universidade Federal de Santa Catarina. Florianópolis: Brazil. 2014, 293p.

GEORGE, A.P.; BROADLEY, R.H.; NISSEN, R.J.; WARD, G. Effects of new rest-breaking chemicals on flowering, shoot production and yield of subtropical tree crops. Acta Horticulturae, The Hague, v.575, p.835-840, 2002. DOI: https://doi.org/10.17660 / ActaHortic.2002.575.99.

HAWERROTH, F.J.; PETRI, J.L.; LEITE, G.B.; HERTER, F.G. Brotação das gemas de macieiras 'Imperial Gala' e 'Fuji Suprema” pelo uso de Erger e Nitrato de cálcio. Rev. Bras. Fruticultura, Jaboticabal, v.32, n.2, p.343-350, 2010. DOI: https://doi.org/10.1590/s010029452010005000074.

HERNÁNDEZ, G; CRAIG, R.L. Effects of alternatives to hydrogen cyanamide on commercial kiwifruit production. Acta Horticulturae, v.913, p.357-363, 2011. DOI: https://doi. org/10.17660 / ActaHortic.2016.1130.19.

PASA, M.S.; FELIPETO, J.; NAVA, G.; SOUZA, A.L.; BRIGHENTI, A.F.; PETRI, J.L. Perfor-» 
Tabela 4. Produção por planta (kg e número de frutos) de macieiras 'Maxi Gala', tratadas com diferentes indutores de brotação, em quatro ciclos - Caçador, SC

Table 4. Production per tree ( $k g$ and number of fruits) of 'Maxi Gala' apple trees, treated with different bud breaking, in four cycles -

Caçador, SC

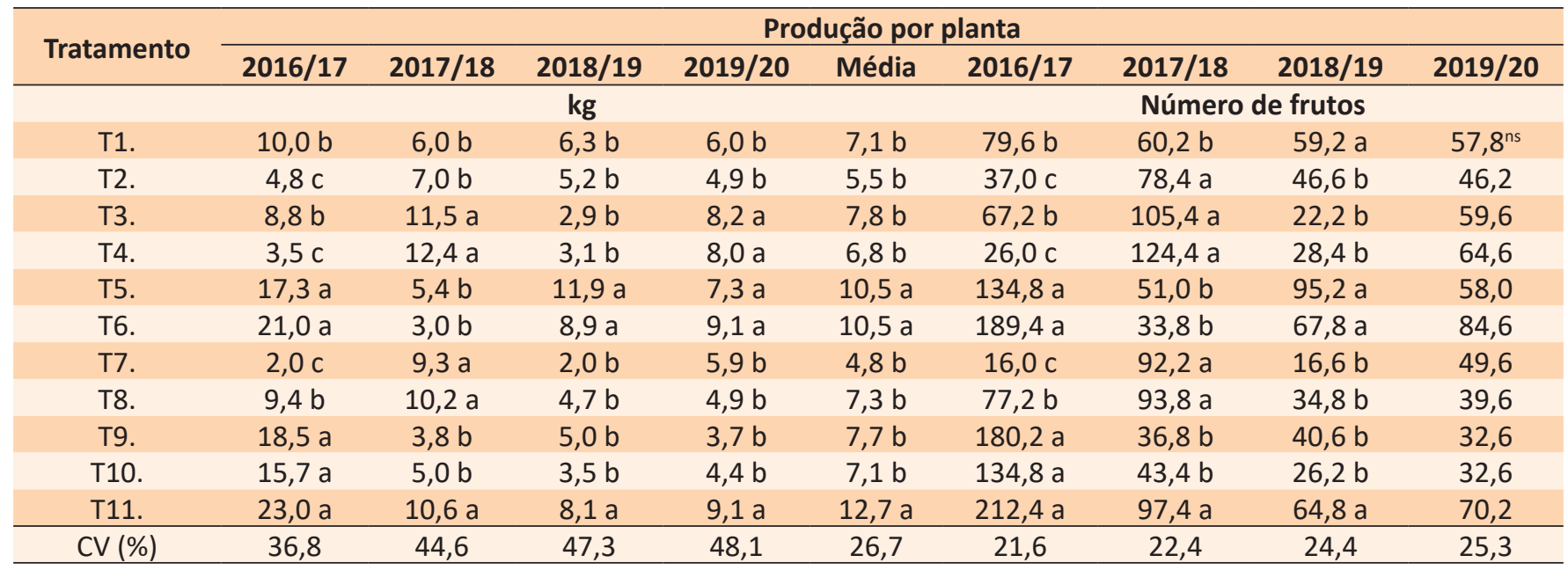

Médias seguidas de mesma letra nas colunas não diferem entre si pelo teste de Scott-Knott a $5 \%$ de probabilidade. ns = não significativo $(p>0,05)$. T1.Testemunha; T2. OM 3,5\% + CH 0,7\% Estádio B; T3. (OM 3,5\% + CH 0,7\% Estádio B) + (OM 3,5\% + CH 0,7\% 7 DA); T4. (OM 3,5\% + CH 0,7\% Estádio B) + (OM 3,5\% + CH 0,7\% 14 DA); T5. (OM 3,5\% + CH 0,7\% Estádio B) + (OM 3,5\% 7 DA); T6. (OM 3,5\% + CH 0,7\% Estádio B) + (OM 3,5\% 14 DA); T7. (OM 3,5\% + CH 0,7\% Estádio B) + (CH 1,0\% 7 DA); T8. (OM 3,5\% + CH 0,7\% Estádio B) + (CH 1,0\% 14 DA); T9. (OM 3,5\% + CH 0,7\% Estádio C) + (CH 0,7\% 5 DA); T10. (OM 3,5\% + CH 0,7\% Estádio C) + (OM 3,5\% + CH 0,7\% $10 \mathrm{DA}) ;$ T11. (OM 3,5\% + CH 0,7\% Estádio C) + (OM 3,5\% 10 DA).

Tabela 5. Massa média de frutos (g) de macieiras 'Maxi Gala', tratadas com diferentes indutores de brotação, em quatro ciclos - Caçador, SC

Table 5. Average fruit mass ( $\mathrm{g}$ ) of 'Maxi Gala' apple trees, treated with different bud breaking, in four cycles - Caçador, SC

\begin{tabular}{cccccc}
\hline \multirow{2}{*}{ Tratamento } & \multicolumn{5}{c}{ Massa média dos frutos } \\
\cline { 2 - 6 } & 2016/17 & $\mathbf{2 0 1 7 / 1 8}$ & $\mathbf{2 0 1 8 / 1 9}$ & $\mathbf{2 0 1 9 / 2 0}$ & Média \\
\hline & & \multicolumn{5}{c}{$\mathbf{g}$} \\
T1. & $128,2 \mathrm{a}$ & $100,8 \mathrm{a}$ & $106,1^{\text {ns }}$ & $106,0 \mathrm{~b}$ & $110,3 \mathrm{a}$ \\
\hline T2. & $130,7 \mathrm{a}$ & $89,4 \mathrm{~b}$ & 110,8 & $101,4 \mathrm{~b}$ & $108,1 \mathrm{~b}$ \\
\hline T3. & $133,3 \mathrm{a}$ & $108,5 \mathrm{a}$ & 132,1 & $138,4 \mathrm{a}$ & $128,1 \mathrm{a}$ \\
\hline T4. & $133,9 \mathrm{a}$ & $100,9 \mathrm{a}$ & 112,4 & $120,8 \mathrm{a}$ & $117,0 \mathrm{a}$ \\
\hline T5. & $129,4 \mathrm{a}$ & $102,6 \mathrm{a}$ & 131,9 & $125,8 \mathrm{a}$ & $122,4 \mathrm{a}$ \\
\hline T6. & $112,9 \mathrm{~b}$ & $89,7 \mathrm{~b}$ & 140,9 & $113,3 \mathrm{~b}$ & $114,2 \mathrm{~b}$ \\
\hline T7. & $128,8 \mathrm{a}$ & $100,4 \mathrm{a}$ & 123,0 & $117,3 \mathrm{~b}$ & $117,4 \mathrm{a}$ \\
\hline T8. & $122,5 \mathrm{a}$ & $108,7 \mathrm{a}$ & 136,9 & $122,7 \mathrm{a}$ & $122,7 \mathrm{a}$ \\
\hline T9. & $103,4 \mathrm{~b}$ & $99,1 \mathrm{a}$ & 122,1 & $114,8 \mathrm{~b}$ & $109,8 \mathrm{a}$ \\
\hline T10. & $116,3 \mathrm{~b}$ & $115,4 \mathrm{a}$ & 133,6 & $133,7 \mathrm{a}$ & $124,7 \mathrm{a}$ \\
\hline T11. & $109,0 \mathrm{~b}$ & $106,2 \mathrm{a}$ & 126,6 & $129,3 \mathrm{a}$ & $117,8 \mathrm{a}$ \\
\hline CV (\%) & 6,8 & 9,1 & 19,3 & 9,5 & 9,1 \\
\hline
\end{tabular}

Médias seguidas de mesma letra nas colunas não diferem entre si pelo teste de Scott-Knott a $5 \%$ de probabilidade. $n s=$ não significativo ( $>>0,05)$. T1.Testemunha; T2. OM 3,5\%+CH 0,7\% Estádio B; T3. (OM $3,5 \%+\mathrm{CH} 0,7 \%$ Estádio B $)+(\mathrm{OM} 3,5 \%+\mathrm{CH} 0,7 \% 7 \mathrm{DA}) ;$ T4. (OM 3,5\% + CH 0,7\% Estádio B) + (OM 3,5\% + CH 0,7\% 14 DA); T5. (OM 3,5\% + CH 0,7\% Estádio B) + (OM 3,5\% 7 DA); T6. (OM 3,5\% + CH 0,7\% Estádio B) + (OM 3,5\% $14 \mathrm{DA}) ; \mathrm{T7}$. (OM 3,5\% + CH 0,7\% Estádio B) + (CH 1,0\% $7 \mathrm{DA}) ; \mathrm{T} 8 .(\mathrm{OM} \mathrm{3,5 \% +CH} \mathrm{0,7 \%}$ Estádio B ) + (CH 1,0\% 14 DA); T9. (OM 3,5\% + CH 0,7\% Estádio C) + (CH 0,7\% 5 DA); T10. (OM 3,5\% + CH 0,7\% Estádio C) + (OM 3,5\% + CH 0,7\% 10 DA); T11. (OM 3,5\% + CH 0,7\% Estádio C) + (OM 3,5\% 10 DA). mance of 'Maxi Gala' apple trees as affected by budbreak promoters, in São Joaquim-SC. Rev. Bras. Fruticultura, Jaboticabal, v. 40, n.5, 2018. DOI: https://doi.org/10.1590/010029452018537.

PETRI, J.L.; HAWERROTH, F.J.; LEITE, G.B.; COUTO, M.; FRANCESCATTO, P. Apple phenology in subtropical climate conditions. In: Xiaoyang Zhang Phenology and Climate Change. Rijeka, Croatia: InTech, v.1, p.195215, 2012.

PETRI, J.L.; LEITE, G.B.; HAWERROTH, F.J. Time of Erger aplication for budbreak induction in apple trees. Acta Horticulturae, Leuven, n.872, p. 205-210, 2010. DOI: https:// doi.org/10.17660 / ActaHortic.2010.872.27.

PETRI, J.L.; LEITE, G.B.; PUTTI, G.L. Apple treebudbreak promoters in mildwinterconditions. Acta Horticulturae, The Hague, v.774, p.291-296, 2008. DOI: https://doi. org/10.17660 / ActaHortic.2008.774.39.

SEZERINO, A.A. Sistema de produção para a cultura da macieira em Santa Catarina. Epagri. 2018. 136p.

UBER, S.C. ; PETRI, J.L.; KRETZSCHMAR, A.A.; FAGUNDES, E. ; CORREA, D.; SILVEIRA, F.N. Alternativas ao uso de cianamida hidrogenada na indução da brotação de gemas em macieiras 'Maxi Gala'. Acta iguazu, Cascavel, v.8, n.3, p. 126-147, 2019. DOI: https://doi. org/10.48075/actaiguaz.v8i3.21087. 